This document is confidential and is proprietary to the American Chemical Society and its authors. Do not copy or disclose without written permission. If you have received this item in error, notify the sender and delete all copies.

\title{
Live follow-up of enzymatic reactions inside the cavities of synthetic giant unilamellar vesicles (GUVs) equipped with membrane proteins mimicking cell architecture
}

\begin{tabular}{|r|l|}
\hline Journal: & ACS Synthetic Biology \\
\hline Manuscript ID & sb-2018-00104c.R1 \\
\hline Manuscript Type: & Article \\
\hline Date Submitted by the Author: & 02-Aug-2018 \\
\hline Complete List of Authors: & $\begin{array}{l}\text { Garni, Martina; University of Basel } \\
\text { Einfalt, Tomaz; University Basel, Chemistry Department } \\
\text { Goers, Roland; University of Basel, Department of Chemistry } \\
\text { Palivan, Cornelia; University of Basel, Chemistry Department } \\
\text { Meier, Wolfgang; University of Basel, Department of Chemistry }\end{array}$ \\
\hline
\end{tabular}




\title{
Live follow-up of enzymatic reactions inside the cavities of synthetic giant
} unilamellar vesicles (GUVs) equipped with membrane proteins mimicking cell architecture

\author{
Martina Garni ${ }^{1}$, Tomaz Einfalt ${ }^{1}$, Roland Goers ${ }^{1,2}$, Cornelia G. Palivan ${ }^{1 *}$, Wolfgang Meier ${ }^{1 *}$ \\ ${ }^{1}$ Department of Chemistry, University of Basel, Mattenstrasse 24a, CH-4002 Basel, Switzerland \\ ${ }^{2}$ Department of Biosystems Science and Engineering, ETH Zürich, Mattenstrasse 26, CH-4058 Basel, \\ Switzerland
}




\section{Abstract}

Compartmentalization of functional biological units, cells and organelles serves as an inspiration for the development of biomimetic materials with unprecedented properties and applications in biosensing and medicine. Due to the complexity of cells, the design of ideal functional materials remains a challenge. An elegant strategy to obtain cell-like compartments as novel materials with bio-functionality is the combination of synthetic micrometer-sized giant unilamellar vesicles (GUVs) with biomolecules because it enables studying the behavior of biomolecules and processes within confined cavities. Here, we introduce a functional cell mimetic compartment by inserting the model biopore bacterial membrane protein $(\mathrm{OmpF})$ in thick synthetic membranes of an artificial GUV compartment that encloses - as a model - the oxidative enzyme horseradish peroxidase. In this manner a simple and robust cell mimic is designed: the biopore serves as a gate, which allows substrates to enter cavities of the GUVs, where they are converted into products by the encapsulated enzyme, and then released in the environments of GUVs. Our bio-equipped GUVs facilitate the control of specific catalytic reactions in confined micro-scale spaces mimicking cell size and architecture and thus provide a straightforward approach serving to obtain deeper insights in the real time of biological processes inside cells.

Keywords: giant unilamellar vesicle, enzyme reaction, membrane protein, synthetic membranes

Eukaryotic cells, as complex assemblies with highly defined structures based on organelles, and a cytoplasm with a variety of biomolecules enclosed by the cellular plasma membrane, serve as an inspiration for the development of new functional systems. ${ }^{1}$ In this respect, the bottom-up approach to mimic the hierarchical organisation and functionality of various cellular structures or even of entire eukaryotic cells ${ }^{2}$ is the focus for producing new functional 
materials with a large variety of applications, such as therapeutics, ${ }^{3}$ catalysis, ${ }^{4}$ biosensing, and surface technology. ${ }^{5}$ Biomimetic materials are created by combining synthetic assemblies with active biomolecules or complexes (proteins, enzymes, DNA). ${ }^{2}$ Self-assembly processes that take place in biological systems can be used to obtain biomimetic materials in which the bio-molecule induces functionality, whilst the supramolecular assemblies (micelles, nanoparticles, tubes, liposomes, polymersomes, planar membranes ${ }^{6,7}$ or micrometer size giant unilamellar vesicles (GUVs)) serve as stable matrices. ${ }^{8}$ The development of functional biomimetic systems requires an understanding of the various reactions that occur within the cellular microenvironment and at cellular interfaces. ${ }^{9,10}$ In this respect, synthetic compartments are very appealing supramolecular assemblies, because their architecture offers well defined and protected reaction spaces for encapsulated catalytic compounds (enzymes, proteins) similar to the compartimentalization of cells or natural organelles. Rudimental cell mimics in the micrometer range known as lipid GUVs are among other used to study lipid membrane permeability ${ }^{11,12}$, membrane protein reconstitution ${ }^{13}$ and confined enzymatic reactions ${ }^{14}$. Polymer compartments (polymersomes with nanometer sizes and synthetic GUVs with micrometer sizes) self-assembled from amphiphilic block copolymers, ${ }^{15,16}$ have become an elegant solution for the development of functional compartments with various advantages over lipid compartments (liposomes and lipid GUVs), such as an improved stability and the huge variety of polymers whose chemistry can be tailored to achieve specific properties (stimuli-responsiveness, flexibility, crystallinity). ${ }^{15}$ Polymersomes have been developed for various applications, such as conventional drug delivery systems, ${ }^{17,18}$ catalytic nanocompartments named nanoreactors, ${ }^{19-21}$ biosensors, ${ }^{22}$ and even artificial organelles that act as simple mimics of cellular organelles. ${ }^{23}$ Interestingly, the study of enzymatic reactions taking place inside polymersomes as confined reaction spaces with sizes less than $300 \mathrm{~nm}$ indicated that in specific conditions it was possible to induce an increase in the bioactivity of encapsulated enzymes, ${ }^{24}$ whilst the presence of crowding agents (i.e. PEG) inside the cavity 
induced a decrease in bio-activity. ${ }^{24}$ Despite the use of polymersomes in various applications, there are still important unanswered questions, such as: How do biomolecules (enzymes, proteins, substrates) behave inside confined compartments with sizes that mimic cells? Is there any interaction between encapsulated compounds and compartment membranes? Do encapsulated compounds diffuse freely inside the cavities, or are they attached to the membrane? If so, does this possible interaction affect the activity and stability of the encapsulated compounds? Development of advanced functional compartments requires detailed responses to such questions in order to understand the complexity and specificity of natural bio-compartments.

To answer such questions, polymer GUVs can serve as a platform that allows a deeper insight into the molecular processes (reactions, interactions) that take place inside confined cavities, in their membranes, and at the interface, by real-time visualization using microscopic techniques. In comparison to GUVs nano-sized polymersomes can only give an indirect insight into membrane protein reconstitution and localization of enzyme reactions. GUVs can be measured directly by established microscopic techniques as their sizes match cell dimensions, and reactions or interactions can now be visualized. Thus, they represent a simple model system for studying the above mentioned processes and interactions in a cell-like manner. In this respect, polymer GUVs are of particular interest because their higher mechanical stability than lipid-based GUVs allows these processes to be studied for longer periods of time. Unlike lipid-based GUVs, there are currently only a few examples of polymer GUVs being used as functional platforms. ${ }^{25}$ For example, synthetic GUVs based on poly(butadiene)- $b$-poly(ethylene oxide) $\left(\mathrm{PBut}_{2.5}-b-\mathrm{PEO}_{1.3}\right)$ loaded with hydrophilic dyes, liposomes (DPPC) and polymersomes $\left(\mathrm{PBut}_{1.2}-b-\mathrm{PEO}_{0.6}\right)$ allowed a fast selectively triggered release due to a light-induced increase in the osmotic pressure, which resulted in rupture of the GUVs. ${ }^{26}$ Despite the potential application of this system for directed delivery and 
controlled local dosing of active compounds, applications of this approach are limited by the fast-irreversible decomposition of the GUVs due to the membrane to volume ratio compared to nano-sized vesicles.

PB- $b$-PEO polymer GUVs can mimic structural and functional eukaryotic cells by encapsulating enzyme-filled intrinsically porous PS- $b$-PIAT polymer nanoreactors with free enzymes and substrates to fulfil a three-enzyme cascade reaction inside the multicompartmentalized structures. ${ }^{27}$ However, that work provided no information about the localization of the enzymes, and only the fluorescence product was observed by spinning disk confocal microscopy. Furthermore, the transmembrane exchange of substrates and products occurred because of the intrinsic permeability of the membrane, and unlike in cell membranes was not mediated by membrane proteins.

Proton transport through polymer polyethylene oxide polybutadiene (PEO-PBD) GUVs membranes has been achieved by incorporating two different synthetic pores, self-assembled from either a dendritic dipeptide or a dendritic ester. However, assembly of dendrimers into stable helical pores was not possible in the case of poly(2-methyloxazoline)-blockpoly(dimethylsiloxane)-block-poly(2-methyloxasoline) (PMOXA-PDMS-PMOXA)-based GUVs, either because of the significant solubility difference between PBD and PDMS, or the differences between di- and triblock architectures. ${ }^{28}$

A more challenging approach for development of cell-mimics based on synthetic GUVs is to insert biopores and membrane proteins into their membranes to induce enhanced permeability to specific ions or molecules, or to support in situ reactions while preserving the 3D architectures of the GUVs. This approach gives the possibility of understanding the biological function of the biopores or membrane proteins, and the visualization of transmembrane processes in real-time. An example is GUVs based on the rendering of PMOXA-PDMSPMOXA triblock copolymer permeable for $\mathrm{Ca}^{2+}$ ions by inserting an unselective ionophore, 
Lasalocid A, and a highly selective ionophore $N, N$-dicyclohexyl- $N^{\prime}, N^{\prime}$-dioctadecyl-3oxapentane-1,5-diamide ${ }^{29}$ whilst insertion of the channel forming peptide alamethicin allowed unselective transport of cations and anions. ${ }^{29}$ The insertion and functionality of the small pore-forming peptide gramicidin (gA) in synthetic PMOXA-PDMS-PMOXA GUVs membranes was visualized in real time by the fluorescence change of the encapsulated dye inside inner cavities. ${ }^{8}$ Interestingly, functional insertion of gA could be achieved in thick membranes up to 5 times greater than the size of gA. ${ }^{8}$ The ability of synthetic membranes, to allow insertion of biopores and membrane proteins (KcsA, OmpF and AqpZ) that are several times smaller than the membrane thickness is the result of its intrinsic fluidity, which overcomes the significant hydrophobic mismatch, if the copolymers are appropriately selected chemically, as in the case of diblock and triblock PMOXA-PDMS-PMOXA copolymers. ${ }^{25,30,31}$ However, to the best of our knowledge, the proof of a functional reconstitution of membrane proteins in synthetic GUVs with cell-like membranes has not yet been established.

Here we present the functional reconstitution of a membrane protein in thick and stable synthetic membranes of GUVs, in order to support cell-like functionality - an enzyme reaction that takes place within a GUV micro-sized cavity (Figure 1). Membrane protein functionality inside GUV membranes represents the key factor for enabling diffusion of the substrates/products of the reaction, and therefore supporting the in situ reaction in a cell-like manner. 
We selected a cysteine double mutant of the bacterial outer membrane porin $\mathrm{F}(\mathrm{OmpF})$ as the model membrane protein, because it has various advantages, such as well-known stability and a pore size, which allows diffusion of molecules up to $600 \mathrm{Da}$. The controlled labeling of the cysteine residues within the OmpF backbone by fluorophores allows the OmpF reconstitution to be monitored. ${ }^{32}$ In addition, OmpF has already been inserted into PMOXA-PDMSPMOXA polymersome membranes with sizes around $200 \mathrm{~nm}$, either as wild type, ${ }^{32}$ or as a mutant, ${ }^{33}$ and demonstrated to allow molecular diffusion. Therefore, we chose this type of amphiphilic copolymer to generate GUVs. The crucial step in our approach was to simultaneously encapsulate an enzyme inside GUVs and insert the OmpF double mutant to 
provide the trans-membrane exchange of substrates and products for the in situ enzymatic reaction. The molecular cut-off of OmpF pores plays a dual role: i) it prevents the model enzyme horseradish peroxidase (HRP) from escaping from the cavity of the GUVs, and ii) it allows diffusion of the substrates $\left(\mathrm{H}_{2} \mathrm{O}_{2}\right.$, Amplex UltraRed (AR)) and the fluorescent product of the enzymatic reaction (resorufin-like product). The reaction has been characterized on whole GUV populations by flow cytometry, whilst conversion into the fluorescent product inside individual GUVs has been detected by confocal laser scanning miscroscopy (CLSM) in real time by time-lapse recording. Real time visualization allows a deep insight regarding the membrane protein and enzyme localization, synthetic membrane permeability, and calculation of the apparent rate constant. Membrane protein functionality in the synthetic membranes of GUVs together with the in situ enzymatic reaction represents a necessary step towards the production of more sophisticated reactions inside functional compartments. Such synthetic GUVs equipped with membrane proteins represent robust functional assemblies that benefit from the stability of the synthetic membrane and the activity of the biomolecules: they open the opportunity of providing detailed insight into the design of multifunctional systems that serve as simple models of artificial cells.

\section{Insertion of the channel protein OmpF}

An essential step towards the production of synthetic GUVs with controlled membrane permeability and a confined space for enzymatic reactions is the reconstitution of the double mutant of the channel porin OmpF, OmpF-M. In order to visualize OmpF-M reconstitution in the $\mathrm{PMOXA}_{7}-\mathrm{PDMS}_{49}-\mathrm{PMOXA}_{7}$ polymer membrane of GUVs by CLSM, $300 \mathrm{mM}$ sucrose was used as a default rehydration solution, since it allows GUVs to settle once they are dispersed in phosphate buffered saline (PBS) $(150 \mathrm{mM}, \mathrm{pH}=7.4)(\mathrm{SI})$. In addition, the 
borosilicate glass surface was plasma-treated to allow GUVs to settle even if the sucrose could diffuse out of the GUV cavities due to the reconstituted OmpF. The cysteine residues replacing the native amino acids $\mathrm{K} 89$ and R270 in OmpF-M were conjugated with a fluorophore (Atto 488 Maleimide), ${ }^{33}$ and non-reacted Atto 488 Maleimide was removed by filtration and dialysis. Atto 488 modification of OmpF-M has been evaluated by fluorescence correlation spectroscopy (FCS) and gel electrophoresis. FCS allows the determination of the diffusion time for fluorescent molecules through the confocal volume of the confocal laser scanning microscope (CLSM), and thus their binding to compounds/assemblies with significantly higher molecular weights by measuring the respective diffusion times ${ }^{34,35}$. Investigating the efficient chemical coupling reaction and successful purification of OmpF is of importance, as free dye molecules could unspecificially stain the polymer giant membranes. By dividing the molecular brightness (counts per molecule, $\mathrm{CPM}$ in $\mathrm{kHz}$ ) of Atto 488 maleimide bound to OmpF-M in $1 \%$ OG PBS $(4.5 \pm 0.3 \mathrm{kHz})$ by Atto 488 in PBS $(3.1 \pm$ $1.23 \mathrm{kHz}$ ), a labeling efficiency of an average of 1.5 Atto 488 molecules per OmpF-M monomer was obtained. No residual fluorescence was observed after purification when Atto 488 was added to wild type OmpF in $3 \%$ OG, or to $3 \%$ OG alone, thus proving that the maleimide fluorophore binds specifically to the cysteine residues of OmpF-M (Figure 2A, Figure S1). 
A

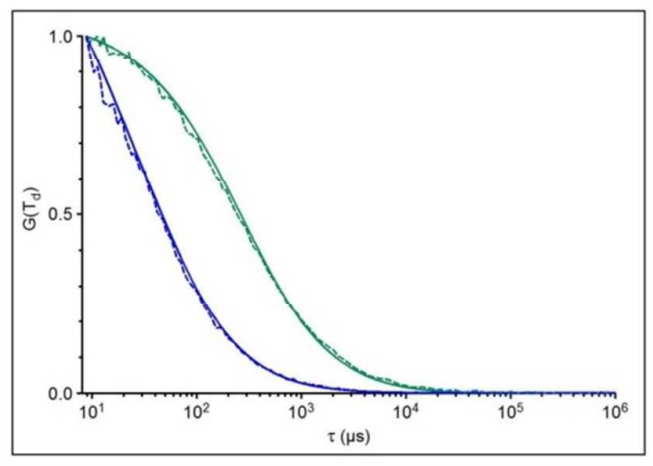

c

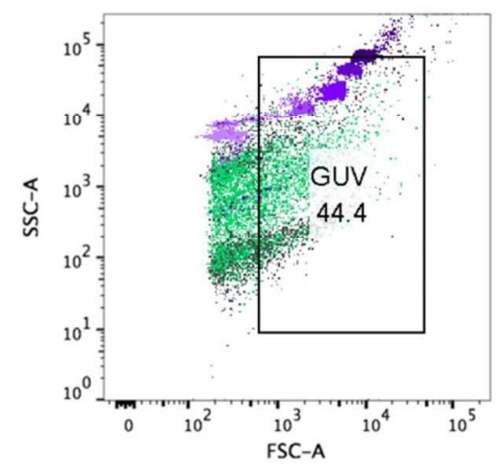

B

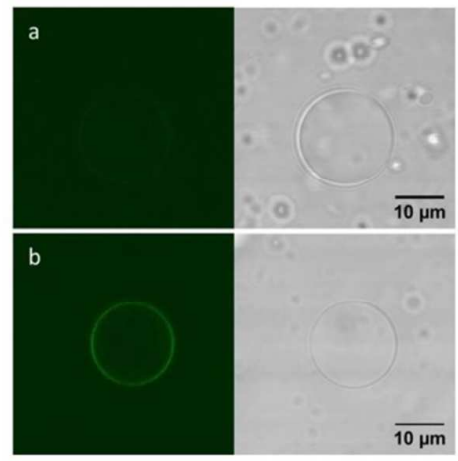

D

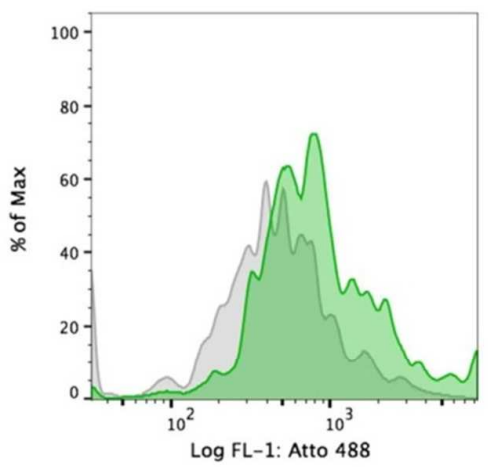

Figure 2. Labeling and reconstitution of OmpF-M. A. FCS autocorrelation curves of Atto 488 in PBS (Blue), and OmpF-Atto 488 in 3\% OG (Green). Dotted line - experimental auto correlation curves, Full line - fit. Curves normalized to 1 to facilitate comparison. B. CLSM micrographs of GUVs: without labeled OmpF-M (a), and with labeled OmpF-M (Atto 488) reconstituted into the synthetic membrane (b). C: Flow cytometry analysis of HRP-loaded GUVS containing OmpF-M (green), HRP loaded GUVs without OmpF-M (dark-gray), and polystyrene latex beads with diameters $2 \mu \mathrm{m}, 3 \mu \mathrm{m}, 5 \mu \mathrm{m}, 7 \mu \mathrm{m}$ s, and $10 \mu \mathrm{m}$ (visualized in increasing size from light purple to dark purple). D: Flow cytometry analysis of PMOXA-PDMS-PMOXA GUVS with OmpF-Atto 488 (green) or without OmpF-Atto 488 (gray).

To determine whether OmpF was inserted in GUV membranes, CLSM measurements were carried out by using GUVs prepared in the presence of Atto 488 labeled OmpF-M (Atto 488OmpF-M), and compared with GUVs in the presence of unlabeled OmpF-M (Figure 2B). Membranes of the GUVs with unlabeled OmpF-M remained dark, whilst those of GUVs with Atto 488-OmpF-M presented a distinct fluorescent membrane (shown in green in Figure 2B), which clearly indicates successful insertion of Atto 488 OmpF-M. The use of film rehydration 
technique for GUV preparation yields a polydisperse GUV population (size, lamellarity) as observed in CLSM and FACS (Figure 2B, 2C), which helped us investigate influence of size on enzyme apparent rate constant. In future studies specific size ranges could be selected and polydispersity's improved by use of droplet transfer and microfluidic approaches ${ }^{36,37}$, however these might not be compatible with the biomolecules due to the use of organic solvents. In addition to CLSM measurements, insertion of Atto 488-OmpF-M was evaluated by flow cytometry, which has previously been used for analysis of lipid GUVs. ${ }^{38}$ Sizes of prepared GUVs were calibrated using the forward scattering (FSC-A) of polystyrene latex beads in the size range of $2-10 \mu \mathrm{m}$ (Figure $2 \mathrm{C}$ ). Taking into account the GUV population between $3-10 \mu \mathrm{m}$, a significant shift in fluorescence intensity was observed for the GUV population with reconstituted Atto 488-OmpF compared to the GUV population without OmpF (Figure 2D, Figure S2). The GUV population smaller than $3 \mu \mathrm{m}$ was discarded in line with CLSM measurements where GUVs in this size range were too small for precise measurements inside their cavities.

\section{Enzyme encapsulation inside the cavities of GUVs}

We were interested in encapsulating enzymes within the cavities of GUVs and analysing how they behave inside a cell-like confined space, lacking the crowded environment of the cytoplasm. In addition, the enzymatic reaction was used to verify diffusion of the substrates through the OmpF-M pores.

In order to follow the encapsulation of the model enzyme horseradish peroxidase (HRP) in GUVs, lysine residues of HRP were conjugated with Atto 488 succinimide ester, and the unreacted fluorophore was removed by dialysis and filtration. The conjugation and purity of the Atto 488-HRP conjugate was established with FCS (Figure 3A) and SDS-PAGE (Figure 
S3). Atto 488 in solution had a typical diffusion time $\tau_{\mathrm{D}}=44.3 \pm 17 \mu$ s and molecular brightness $(\mathrm{CPM})$ of $4.4 \pm 0.3 \mathrm{kHz}$, whereas the Atto 488 -HRP presented a significantly longer diffusion time $\tau_{\mathrm{D}}=252 \pm 43 \mu \mathrm{s}$ with a molecular brightness $(\mathrm{CPM})$ of $3.4 \pm 0.5 \mathrm{kHz}$. The free fraction of Atto 488 in the HRP-Atto 488 solution was determined with a two component fit of the autocorrelation curve to be $15 \% \pm 11 \%$ (Figure $3 \mathrm{~A}$ ).

A

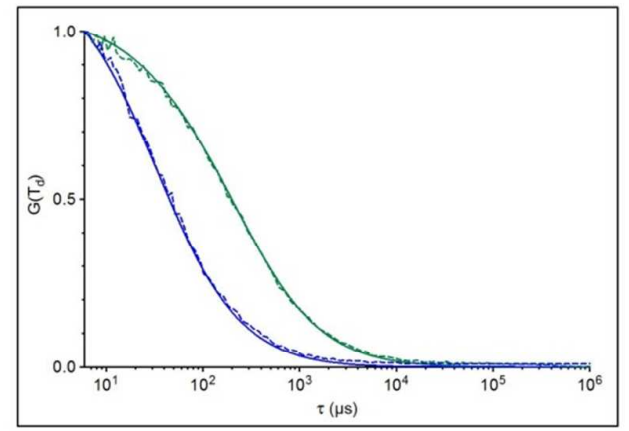

C

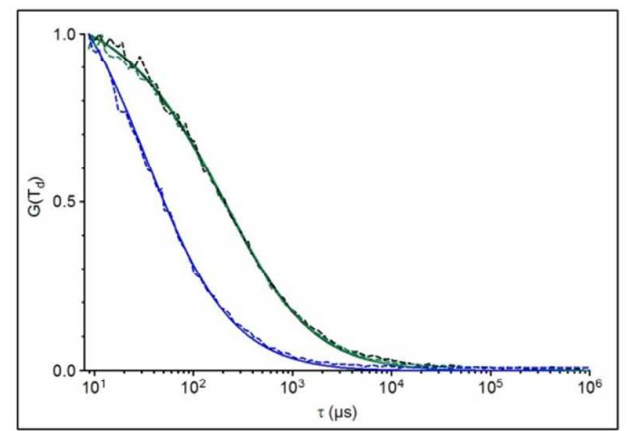

B

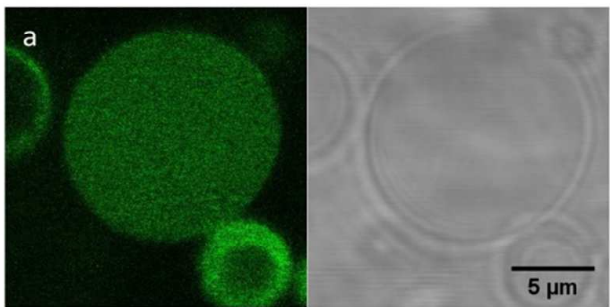

b

$\overline{5 \mu \mathrm{m}}$

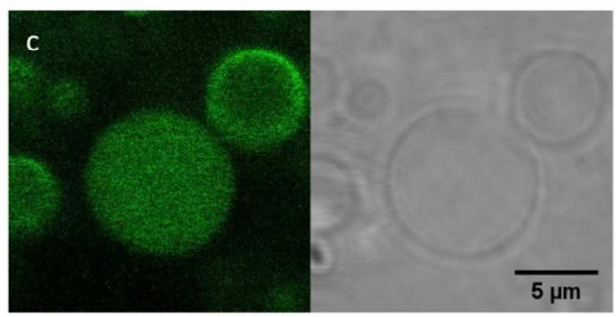

Figure 3. A: FCS autocorrelation curves for Atto 488 in sucrose (Blue), and HRP-Atto 488 in sucrose (Green). Dotted line - experimental auto correlation curves, Full line - fit. Curves are normalized to 1 to facilitate comparison. B: a,b,c: CLSM images of polymer GUVS with labeled HRP (Atto 488) encapsulated. In each image: left: fluorescence channel, right: transmission channel. C: FCS autocorrelation curves for Atto 488 in sucrose (Blue), HRP-Atto 488 in sucrose (Black), HRP-Atto 488 in GUV (Green). Dotted line - experimental auto correlation curves, Full line - fit. Curves normalized to 1 to facilitate comparison.

After encapsulation of Atto 488-HRP in 300mM sucrose solution, and settling of the GUVs to the imaging chamber surface (SI), most of the GUVs measured by CLSM showed 
fluorescence inside their cavities (Figure 3B), indicating successful HRP encapsulation and free diffusion of the enzyme. Not all GUVs had the same amount of HRP encapsulated inside their cavities when observed by CLSM. Due to the nature of the film rehydration method used for the preparation of GUVs different localizations of HRP-Atto488 are expected. In addition, GUV membranes presented only low fluorescence intensity, due to the reflection of fluorescent light between the GUV membrane / cavity interface, and a minor enzymemembrane interaction. No fluorescence was observed outside the GUVs, which demonstrates successful purification of the GUVs after enzyme encapsulation. As self-assembly of the GUVs with encapsulated enzyme is a statistical process, meaning not all GUVs have the same amount of Atto 488-HRP present inside their cavities.

To visualize in more detail, the dynamics of enzyme encapsulation in the GUVs, we performed FCS measurements inside the cavity of Atto 488 labeled HRP loaded GUVs (Figure 3C). As the encapsulated HRP molecules have similar diffusion times (273 $\pm 144 \mu \mathrm{s})$ to the free HRP in sucrose $(252 \pm 43 \mu \mathrm{s})$ resulting in an overlap of their curves shown in green and black (Figure 3C green and black curve), it confirms a successful enzyme encapsulation (at least 10 GUVs were measured by FCS), and no change in the dynamics of the enzyme inside the confined space of the GUVs (Figure 3C).

\section{Enzyme reaction inside GUVs cavities}

To address the question of enzyme functionality within GUV cavities, the rate of oxidation of Amplex UltraRed by HRP in the presence of $\mathrm{H}_{2} \mathrm{O}_{2}$ was first studied in solution by fluorescence spectroscopy and the enzyme activity for HRP in PBS compared to that for HRP in $300 \mathrm{mM}$ sucrose, which was used for GUV formation (Figure S4). There was no evident difference between the two media. Furthermore, without $\mathrm{H}_{2} \mathrm{O}_{2}$ as a co-substrate, there was 
barely any conversion of Amplex UltraRed into the fluorescent resorufin-like product. Similarly, there was no substrate conversion and therefore no fluorescence signal if no substrate Amplex UltraRed or no enzyme was present in solution (Figure S4).

This model enzyme reaction has been recently studied in synthetic compartments with sizes in the nanometer range, namely polymersomes equipped with OmpF-M. ${ }^{33}$ As we modified this method for the formation of OmpF-M equipped polymersomes by using sucrose solution for GUVs preparation instead of PBS, and the addition of OmpF-M in bidistilled water instead of PBS (since the presence of salts disturbed the GUV formation), we were interested in determining whether these modifications affect the OmpF-M reconstitution and further enzymatic reactions. Therefore, we compared the enzymatic reaction inside polymersomes under the above mentioned conditions used for GUVs with those previously reported for polymersomes by fluorescence spectroscopy. ${ }^{32,33}$ For this, OmpF equipped polymersomes loaded with HRP were prepared and characterized (Figure S5, S6). As the in situ enzymatic reaction was not affected, this result indicates that the sucrose solution together with the addition of the OmpF-M in bidistilled water used for the GUVs experiments (see below) does not affect the functional reconstitution of OmpF or the activity of the encapsulated enzyme within polymersomes (Figure S7). This information tells us that the OmpF-M reconstitution together with the enzyme reaction should not be hindered by the conditions used for preparing the GUVs.

By monitoring the enzymatic reaction by CLSM for up to $12 \mathrm{~min}$, it was demonstrated that the membrane protein OmpF is fully functional in the synthetic membrane of GUVs and that it renders the membrane permeable to the substrates and products. We produced videos with an image recording time of 15.7 s/image and $1 \mathrm{~s}$ delay (Video S1 and S2). To establish a starting point $(\mathrm{t}=0)$, the first image was recorded before addition of the substrates. Then, the enzymatic reaction was initiated by adding substrates (Amplex UltraRed and $\mathrm{H}_{2} \mathrm{O}_{2}$ ) to the 
external solution, and the increase in the fluorescent signal associated with the product of the reaction was visualized by CLSM (Figure 4). The same procedure was carried out for HRPloaded GUVs without inserted OmpF-M.

GUV with reconstituted OmpF

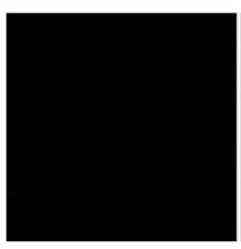

$0 \min$

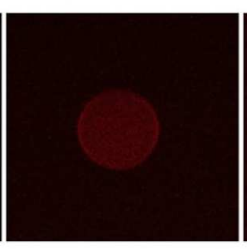

$1 \mathrm{~min}$

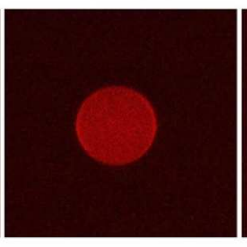

$2 \min$

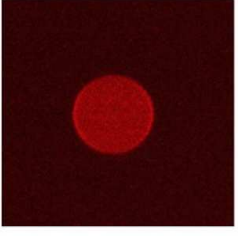

$3 \min$

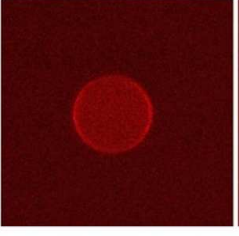

$6 \mathrm{~min}$

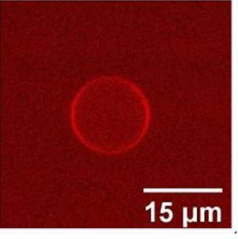

$12 \mathrm{~min}$

GUV without reconstituted OmpF

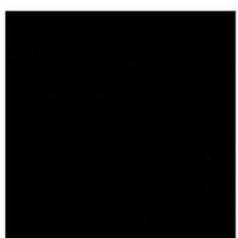

$0 \mathrm{~min}$

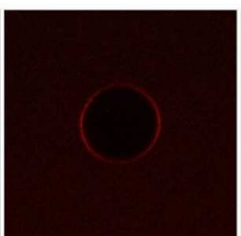

$1 \mathrm{~min}$

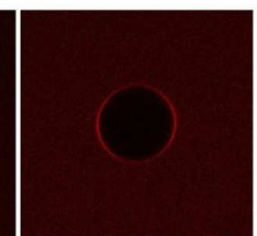

$2 \min$

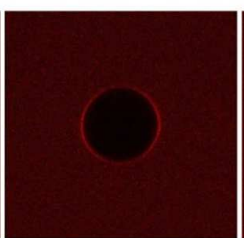

$3 \min$

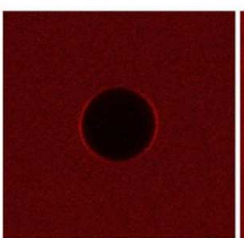

$6 \min$

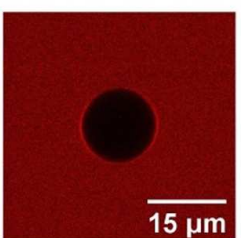

$12 \mathrm{~min}$

Figure 4. CLSM Fluorescence micrographs of single GUVs obtained over time, by recording images at certain time points after initial addition of the substrates $\mathrm{H}_{2} \mathrm{O}_{2}$ and Amplex UltraRed. Top) GUV with reconstituted OmpF-M. Bottom) GUV without OmpF-M.

The increase in the fluorescence intensity within GUVs equipped with OmpF-M results from the formation of the fluorescent resorufin-like product, and proves the functional insertion of OmpF-M that permits molecular transport through the synthetic membrane of the GUVs. On the contrary, there was no increase in fluorescence intensity inside GUVs without inserted OmpF-M, since the substrates, especially the Amplex UltraRed, were unable to pass through the synthetic membrane. Over time, the fluorescence inside the cavity of the OmpF-equipped GUVs decreased slightly (see the change from $3 \mathrm{~min}$ to $6 \mathrm{~min}$ in Figure 4), because the resorufin-like product of the enzyme reaction diffuses out of the GUVs through the OmpF-M pores. As expected, after a certain time, the fluorescence inside the cavity of OmpF-equipped 
GUVs becomes equal to the fluorescence intensity of the environment of the GUVs, which is not the case for GUVs without OmpF-M, where there was almost no fluorescence signal inside the GUVs (dark core of GUVs). Both GUVs with OmpF-M inserted and without OmpF showed a fluorescent membrane over time due to small amounts of the resorufin-like product interacting with GUV membranes. In addition, a second factor, which causes the membrane to appear fluorescent, is the scattering and reflection of fluorescent light between the GUV membrane / cavity interface. Note, that in the following data assessment, the fluorescence intensity of the membrane of the GUVs has not been taken into account. The passive accumulation of the fluorescent resorufin-like product in the background was used as a criterion to determine whether the enzyme reaction has taken place within the cavity of the GUVs. The reaction occurred only if the fluorescence intensity was higher inside the GUVs than in the background. Both phenomena were considered in the data assessment, and consequently the membrane fluorescence was disregarded and the fluorescence intensity of the background was subtracted.

For each image of the sequences, the mean fluorescence intensity of a region of interest (ROI), depending on the individual size of the GUVs, both inside the cavity of each GUV (Figure S8) and outside the GUVs (background) was calculated using the program ImageJ. The mean fluorescence intensity values of the background over time were subtracted from the values inside GUVs cavity (Figure 5 A and C, Figure S8 and S9). Around 70\% of the GUVs with reconstituted OmpF-M showed fluorescence higher than the background inside their cavities indicating that the enzyme reaction occurred, whereas the fluorescence intensity inside the cavity of $\sim 70 \%$ of the GUVs without OmpF showed less fluorescence intensity than the background (Table S1). The reason why some GUVs are not working as expected can be the inhomogeneous encapsulation of the enzyme, membrane defects and different amounts of reconstituted $\mathrm{OmpF}$, resulting from the film rehydration technique. However 
despite the limitations of the film rehydration technique, we observed that if the OmpF is reconstituted the cavities of these GUVs shore a higher fluorescence activity compared to the GUVs without OmpF.

In order to estimate the apparent rate constant, $\mathrm{k}_{\mathrm{app}}$, for the GUVs with and without OmpF, a linear regression of the fluorescence increase within the first 2 min was performed on all the curves, where each curve corresponded to a single GUV with background fluorescence intensity subtracted. Only GUVs with fluorescence higher than the background in case of the OmpF-M equipped GUVs (70\%) were taken into account and vice versa for the GUVs containing no OmpF.

A apparent rate constant of $\mathrm{k}_{\mathrm{app}} \approx 0.011 \mathrm{~s}^{-1}$ was obtained for GUVs with reconstituted OmpF$\mathrm{M}$ (Figure $5 \mathrm{~B}$ ). In contrast, for GUVs without OmpF the negative apparent rate constant was an order of magnitude lower with $\mathrm{k}_{\mathrm{app}} \approx-0.0036 \mathrm{~s}^{-1}$ (Figure $5 \mathrm{D}$ ). The increase in the relative fluorescence intensity for OmpF-equipped GUVs clearly indicates successful in situ bioactivity of HRP (Figure 5 A and B), whilst the decrease towards negative values of the curve for the GUVs without $\mathrm{OmpF}$ is due to an increase in the fluorescence intensity of the background that results from the HRP activity at the exterior of the GUVs (Figure $5 \mathrm{C}$ and D). 
GUVs with reconstituted OmpF

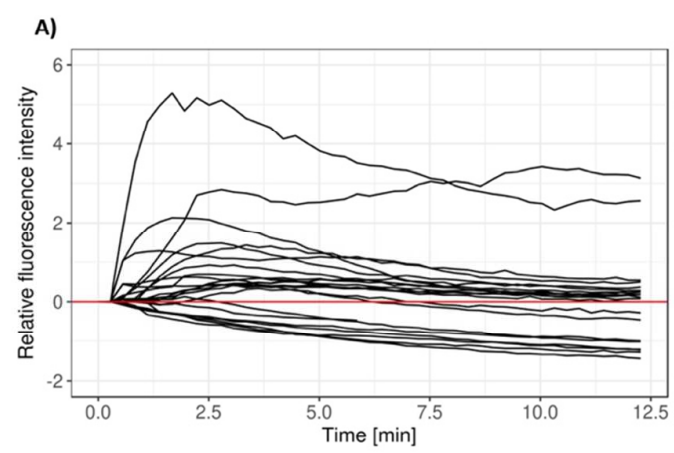

GUVs without reconstituted OmpF

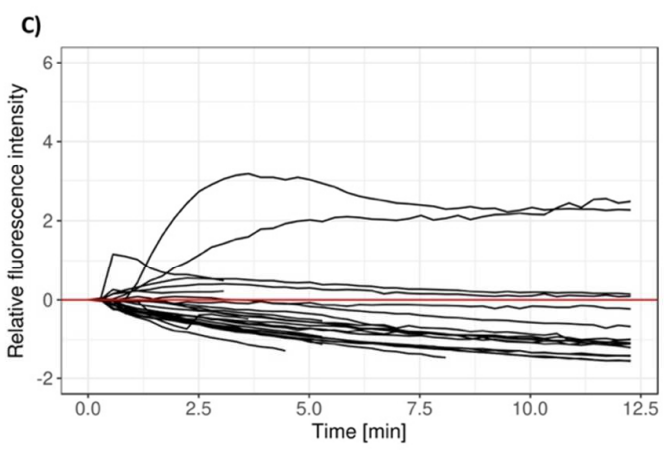

B)

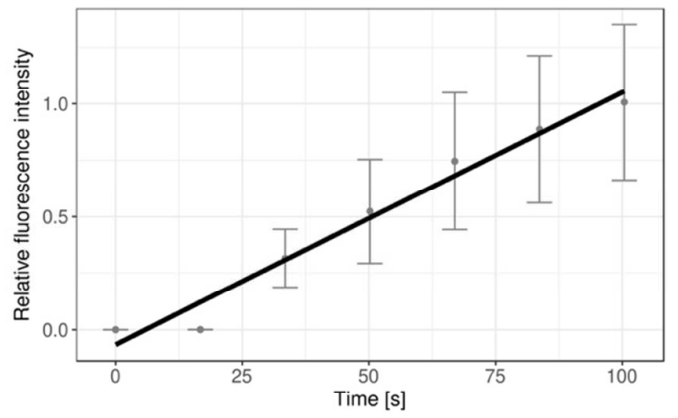

D)

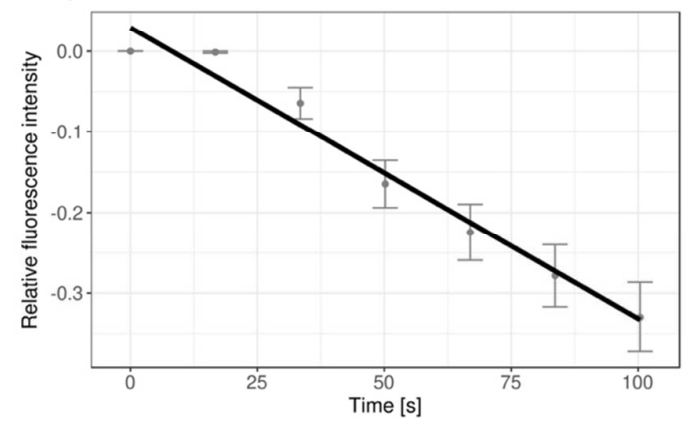

Figure 5. Top: GUVS with reconstituted OmpF-M. Bottom: GUVS without reconstituted OmpF-M A and C: Temporal variation in the relative fluorescence intensity of the resorufin-like product inside the cavities of single GUVS after addition of the reagents $\mathrm{H}_{2} \mathrm{O}_{2}$ and Amplex UltraRed after background subtraction. Each curve represents one GUV. B and D): Linear regressions of change of the fluorescence intensity over time. Shown are the mean values with standard error of the mean (sem). $n=18-25$.

To gain further information on the membrane permeabilization by insertion of the OmpF-M pore, the Amplex UltraRed enzymatic assay was applied in combination with flow cytometry analysis. Oxidation of Amplex UltraRed was observed inside the cavities of HRP loaded GUVs equipped with OmpF-M, in agreement with the results obtained by CLSM, and the significant shift of the fluorescence for HRP loaded GUVs equipped with OmpF-M compared to HRP loaded GUVs without OmpF-M clearly proved that the porins allow substrates to efficiently diffuse into the cavities of the GUVs (Figure 6, Figure S10). 


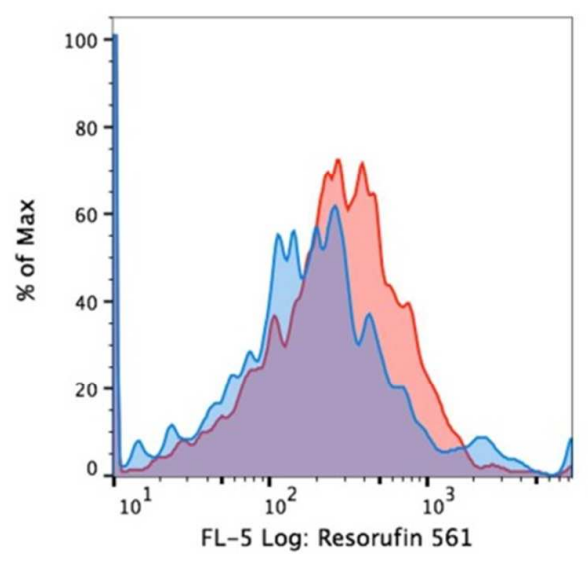

Figure 6. Flow cytometry analysis - Amplex UltraRed enzymatic assay of OmpF equipped (red) or unequipped PMOXA $A_{7}-P D M S_{49}-P M O X A_{7}$ GUVS (blue).

Intensities measured by FACS and CLSM are not directly comparable. FACS represents the average fluorescence intensity of all measured GUVs whereas CLSM images represent only a part of the whole sample, where our criteria were unilamellar GUVs above $5 \mu \mathrm{m}$ in diameter. Not all GUVs observed by CLSM work as expected as described above. FACS represents an average intensity of the whole sample population, hence the difference in signal intensity between FACS and CLSM is expected but the trend observed by both techniques is the same.

Regarding the enzymatic reaction by using CLSM videos over time were recorded and the fluorescence activity over time measured for single GUVs directly after the addition of the substrates. In contrast FACS measurements are based on the flow-through principle. This means the whole GUV population with the added substrates is measured and the fluorescence intensities of each GUV at different time points are averaged during the measuring. Due to the diffusion of the substrates and the product in and out of the GUVs it is challenging to measure the point where the fluorescence of each GUV is highest. 
However despite the difference in measuring methods, the twofold increase of the relative fluorescence intensity in CLSM is comparable to the twofold shift observed in FACS.

Thus these CLSM and flow cytometry results confirm the necessity of functional membrane protein reconstitution inside polymer GUV membranes for production of the fluorescent product by the enzyme confined inside the GUV cavities.

\section{GUV size distribution}

Next, we were interested in assessing whether the size of GUVs affects the enzymatic reaction that takes place within their cavities. To determine the corresponding sizes of the GUVs for a comparison between the dataset from GUVs with reconstituted OmpF-M and GUVs without OmpF-M, it was necessary to investigate whether these two populations showed size differences. First, the diameters of GUVs (both OmpF-equipped GUVs, and GUVs without OmpF) were measured using ImageJ and plotted (Figure S11); a Welch Two Sample t-test showed no significant difference between the OmpF-equipped GUVs and GUVs without OmpF (p-value $=0.27, \alpha=0.05)($ Figure S12).

To carry out a more detailed comparison, the fluorescence intensity of each individual vesicle was taken 260 seconds after the reaction was initiated to ensure a stable signal. A significant difference in fluorescence intensity was observed between the averages of OmpF-equipped GUVs and GUVs without OmpF (Figure S13 A) $(\mathrm{p} \approx 0.024(\alpha=0.05))$.

Further details of the in situ enzymatic reaction were revealed by dividing the GUVs into 4 groups, namely from 6 to $9 \mu \mathrm{m}(\mathrm{A}), 9$ to $11 \mu \mathrm{m}(\mathrm{B}), 11$ to $13 \mu \mathrm{m}(\mathrm{C})$ and 13 to $16 \mu \mathrm{m}(\mathrm{D})$ (Figure S13 B). The difference in intensity of the two GUV populations was less pronounced in the range of 6 to $9 \mu \mathrm{m}$, but the median intensity differed clearly between OmpF equipped 
1

2

3

4

5

6

7

8

9

GUVs and non OmpF equipped GUVs within the size ranges 9 to 11,11 to 13 and 13 to 16 $\mu \mathrm{m}$, and was most pronounced with the higher sizes. In addition to CLSM analysis increasing enzymatic activity in respect to increasing GUV size was also confirmed by flow cytometry (Figure S14). This is in good agreement with the expectation that larger GUVs should contain more enzyme molecules inside their cavities leading to a higher substrate conversion and thus have a higher overall intensity. Even though a slight decrease was observed from group $\mathrm{C}$ to group $\mathrm{D}$, it was not statistically significant $(\mathrm{p} \approx 0.344)$.
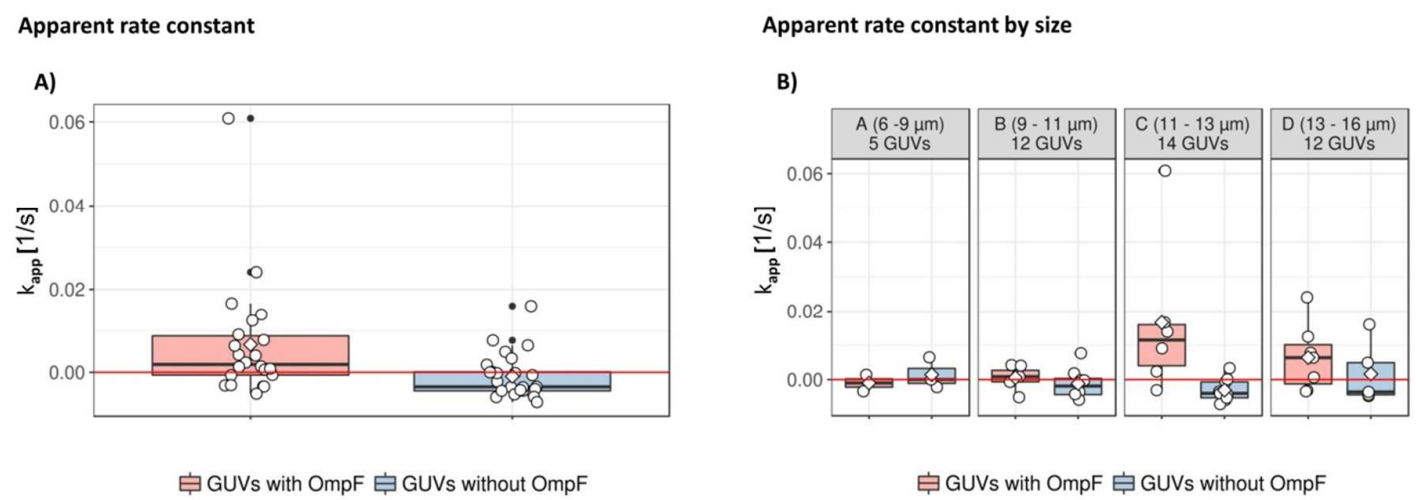

Figure 7. A) Apparent rate constant, $\mathrm{k}_{\mathrm{app}}$, of two populations of GUVs with and without reconstituted OmpF-M. Each GUV was fitted independently. B) Variation of apparent rate constant, $\mathrm{k}_{\mathrm{app}}$, with GUV diameters. The black line $=$ median, white square $=$ average value, white points $=$ data points.

The apparent rate constant, was also calculated for each individual GUV. Again, there were significant differences between GUVs with and those without inserted OmpF (Welch Two Sample t-test, $\mathrm{p}$-value $\approx 0.025, \alpha=0.05$ ) (Figure 7A). Classification of GUVs into the same size clusters as above shows a similar behavior to that described in the previous paragraph (Figure 7B). Overall, the difference in the median apparent rate constant, $\mathrm{k}_{\mathrm{app}}$, is marginal for group A, but there is an obvious difference in group B, and this increased in groups C and D. Since there was a constant enzyme concentration in the rehydration solution, the number of 
enzymes present in a GUV's cavity should depend only on its size, and therefore volume. Thus, with increasing GUV sizes, the volume and the number of enzymes per cavity should increase, and explains the lower apparent rate constant that was observed in group A. Furthermore, larger sizes have more membrane surface, which allows a higher number of OmpF pores to be present. This then results in increased substrate diffusion rates, and a steady stream of substrates and product can be achieved. It appears that the closer the physical characteristics (e.g. size and volume) are to a natural cell, the higher the performance of the system.

This is the first time that a membrane protein $(\mathrm{OmpF})$ has been reconstituted into synthetic polymer membranes of giant unilamellar vesicles (GUVs) with preserved functionality, and is instrumental in initiating an in situ enzymatic reaction by allowing diffusion of substrates and products. The model enzyme reaction inside the GUV cavities was monitored in real-time and gave insight into both the substrate apparent rate constant by the encapsulated enzyme and the reconstitution of the functional membrane protein $(\mathrm{OmpF})$ in synthetic GUV membranes. The large cavities of GUVs allowed free diffusion of the encapsulated enzyme, as proven by FCS. Furthermore, there appears to be a size-dependent trend in enzyme apparent rate constant in respect of GUV diameters. Also, whereas direct observation of internal reactions in established nano-compartment platforms, such as liposomes and polymersomes, are limited by the resolution barrier, GUVs provide a unique opportunity for observing the behavior of biomolecules within defined confined spaces. The combination of biomolecules and synthetic materials found in membrane protein-equipped polymer GUVs not only offers the advantages of increased stability and lower membrane permeability than lipid GUVs, but together with an encapsulated enzyme makes the micro scale compartment platform relevant for various 
applications, as for example a research tool to study biomolecule behavior within these celllike compartments or as biosensors.

Our simultaneous encapsulation and insertion of biomolecules in GUVs allows a thorough understanding of biological processes in real time, and represents a useful platform to study reactions inside confined spaces or interactions of biomolecules with membranes by a straightforward change of enzyme type. As GUVs have sizes similar to those of cells, they allow the relevant study of bio-processes in vitro in a simple manner. Thinking even further ahead, molecular factories and advanced cell mimics could be developed by encapsulating in cell-sized GUVs various polymersomes loaded with different types of enzyme inside their cavities.

\section{Author contributions}

M.G. contributed to the OmpF modification and characterisation, purification GUVs production and characterisation, enzymatic assays, CLSM, flow cytometry, FCS and the writing of the manuscript; T.E. contributed to the OmpF and HRP characterisation and modification, FCS and flow cytometry; R.G. contributed to the enzymatic data evaluation; W.M. and C.G.P contributed enzymatic GUV concept and writing of the manuscript.

\section{Acknowledgments}

We gratefully acknowledge the financial support provided by the Swiss Nanoscience Institute, the Swiss National Science Foundation, and the National Centre of Competence in Research Molecular Systems Engineering. Authors thank S. Lörcher (University of Basel) for synthesizing the polymer. M.G. thanks G. Persy (University of Basel) for TEMmeasurements. Dr. B.A. Goodman for editing the manuscript. 
References:

(1) Rafelski, S. M.; Marshall, W. F. Building the Cell: Design Principles of Cellular Architecture. Nat. Rev. Mol. Cell Biol. 2008, 9 (8), 593.

(2) Salehi-Reyhani, A.; Ces, O.; Elani, Y. Artificial Cell Mimics as Simplified Models for the Study of Cell Biology. Exp. Biol. Med. 2017, 242 (13), 1309-1317.

(3) Sueyoshi, D.; Anraku, Y.; Komatsu, T.; Urano, Y.; Kataoka, K. Enzyme-Loaded Polyion Complex Vesicles as in Vivo Nanoreactors Working Sustainably under the Blood Circulation: Characterization and Functional Evaluation. Biomacromolecules 2017, 18 (4), 1189-1196.

(4) Dinu, M. V.; Spulber, M.; Renggli, K.; Wu, D.; Monnier, C. A.; Petri-Fink, A.; Bruns, N. Filling Polymersomes with Polymers by Peroxidase-Catalyzed Atom Transfer Radical Polymerization. Macromol. Rapid Commun. 2015, 36 (6), 507-514.

(5) Zhang, X.; Lomora, M.; Einfalt, T.; Meier, W.; Klein, N.; Schneider, D.; Palivan, C. G. Active Surfaces Engineered by Immobilizing Protein-Polymer Nanoreactors for Selectively Detecting Sugar Alcohols. Biomaterials 2016, 89, 79-88.

(6) Kowal, J.; Zhang, X.; Dinu, I. A.; Palivan, C. G.; Meier, W. Planar Biomimetic Membranes Based on Amphiphilic Block Copolymers. ACS Macro Lett. 2014, 3 (1), 59-63.

(7) Letchford, K.; Burt, H. A Review of the Formation and Classification of Amphiphilic Block Copolymer Nanoparticulate Structures: Micelles, Nanospheres, Nanocapsules and Polymersomes. Eur. J. Pharm. Biopharm. Off. J. Arbeitsgemeinschaft Pharm. Verfahrenstechnik EV 2007, 65 (3), 259-269.

(8) Lomora, M.; Garni, M.; Itel, F.; Tanner, P.; Spulber, M.; Palivan, C. G. Polymersomes with Engineered Ion Selective Permeability as Stimuli-Responsive Nanocompartments with Preserved Architecture. Biomaterials 2015, 53 (Supplement C), 406-414.

(9) Küchler, A.; Yoshimoto, M.; Luginbühl, S.; Mavelli, F.; Walde, P. Enzymatic Reactions in Confined Environments. Nat. Nanotechnol. 2016, 11 (5), 409.

(10) Garni, M.; Einfalt, T.; Lomora, M.; Car, A.; Meier, W.; Palivan, C. G. Artificial Organelles: Reactions inside Protein-Polymer Supramolecular Assemblies. Chim. Int. J. Chem. 2016, 70 (6), 424-427.

(11) Yoshitani, T.; Yamazaki, M. Water Permeability of Lipid Membranes of GUVs and Its Dependence on Actin Cytoskeletons inside the GUVs. In 2008 International Symposium on Micro-NanoMechatronics and Human Science; 2008; pp 130-134.

(12) Li, S.; Hu, P. C.; Malmstadt, N. Imaging Molecular Transport across Lipid Bilayers. Biophys. J. 2011, 101 (3), 700-708.

(13) Jørgensen, I. L.; Kemmer, G. C.; Pomorski, T. G. Membrane Protein Reconstitution into Giant Unilamellar Vesicles: A Review on Current Techniques. Eur. Biophys. J. 2017, 46 (2), 103-119.

(14) Trantidou, T.; Friddin, M.; Elani, Y.; Brooks, N. J.; Law, R. V.; Seddon, J. M.; Ces, O. Engineering Compartmentalized Biomimetic Micro- and Nanocontainers. ACS Nano 2017, 11 (7), 6549-6565.

(15) Discher, D. E.; Eisenberg, A. Polymer Vesicles. Science 2002, 297 (5583), 967-973.

(16) Discher, B. M.; Won, Y.-Y.; Ege, D. S.; Lee, J. C.-M.; Bates, F. S.; Discher, D. E.; Hammer, D. A. Polymersomes: Tough Vesicles Made from Diblock Copolymers. Science 1999, 284 (5417), 1143-1146.

(17) Pawar, P. V.; Gohil, S. V.; Jain, J. P.; Kumar, N. Functionalized Polymersomes for Biomedical Applications. Polym. Chem. 2013, 4 (11), 3160-3176.

(18) Onaca, O.; Enea, R.; Hughes, D. W.; Meier, W. Stimuli-Responsive Polymersomes as Nanocarriers for Drug and Gene Delivery. Macromol. Biosci. 2009, 9 (2), 129-139. 
(19) Dobrunz, D.; Toma, A. C.; Tanner, P.; Pfohl, T.; Palivan, C. G. Polymer Nanoreactors with Dual Functionality: Simultaneous Detoxification of Peroxynitrite and Oxygen Transport. Langmuir 2012, 28 (45), 15889-15899.

(20) Graff, A.; Winterhalter, M.; Meier, W. Nanoreactors from Polymer-Stabilized Liposomes. Langmuir 2001, 17 (3), 919-923.

(21) Nardin, C.; Thoeni, S.; Widmer, J.; Winterhalter, M.; Meier, W. Nanoreactors Based on (Polymerized) ABA-Triblock Copolymer Vesicles. Chem. Commun. 2000, 0 (15), 1433-1434.

(22) M. Kuiper, S.; Nallani, M.; M. Vriezema, D.; M. Cornelissen, J. J. L.; Hest, J. C. M. van; M. Nolte, R. J.; E. Rowan, A. Enzymes Containing Porous Polymersomes as Nano Reaction Vessels for Cascade Reactions. Org. Biomol. Chem. 2008, 6 (23), 4315-4318.

(23) Tanner, P.; Balasubramanian, V.; Palivan, C. G. Aiding Nature's Organelles: Artificial Peroxisomes Play Their Role. Nano Lett. 2013, 13 (6), 2875-2883.

(24) Baumann, P.; Spulber, M.; Fischer, O.; Car, A.; Meier, W. Investigation of Horseradish Peroxidase Kinetics in an "Organelle-Like" Environment. Small 2017, 13 (17), n/a-n/a.

(25) Xiao, Q.; Yadavalli, S. S.; Zhang, S.; Sherman, S. E.; Fiorin, E.; Silva, L. da; Wilson, D. A.; Hammer, D. A.; André, S.; Gabius, H.-J.; et al. Bioactive Cell-like Hybrids Coassembled from (Glyco)Dendrimersomes with Bacterial Membranes. Proc. Natl. Acad. Sci. 2016, 113 (9), E1134-E1141.

(26) Peyret, A.; Ibarboure, E.; Tron, A.; Beauté, L.; Rust, R.; Sandre, O.; McClenaghan, N. D.; Lecommandoux, S. Polymersome Popping by Light-Induced Osmotic Shock under Temporal, Spatial, and Spectral Control. Angew. Chem. Int. Ed. 2017, 56 (6), 15661570.

(27) Peters, R. J. R. W.; Marguet, M.; Marais, S.; Fraaije, M. W.; van Hest, J. C. M.; Lecommandoux, S. Cascade Reactions in Multicompartmentalized Polymersomes. Angew. Chem. Int. Ed. 2014, 53 (1), 146-150.

(28) Kim, A. J.; Kaucher, M. S.; Davis, K. P.; Peterca, M.; Imam, M. R.; Christian, N. A.; Levine, D. H.; Bates, F. S.; Percec, V.; Hammer, D. A. Proton Transport from Dendritic Helical-Pore-Incorporated Polymersomes. Adv. Funct. Mater. 2009, 19 (18), 2930-2936.

(29) Sauer, M.; Haefele, T.; Graff, A.; Nardin, C.; Meier, W. Ion-Carrier Controlled Precipitation of Calcium Phosphate in Giant ABA Triblock Copolymer Vesicles. Chem. Commun. 2001, 0 (23), 2452-2453.

(30) Itel, F.; Chami, M.; Najer, A.; Lörcher, S.; Wu, D.; Dinu, I. A.; Meier, W. Molecular Organization and Dynamics in Polymersome Membranes: A Lateral Diffusion Study. Macromolecules 2014, 47 (21), 7588-7596.

(31) Itel, F.; Najer, A.; Palivan, C. G.; Meier, W. Dynamics of Membrane Proteins within Synthetic Polymer Membranes with Large Hydrophobic Mismatch. Nano Lett. 2015, 15 (6), 3871-3878.

(32) Einfalt, T.; Goers, R.; Dinu, I. A.; Najer, A.; Spulber, M.; Onaca-Fischer, O.; Palivan, C. G. Stimuli-Triggered Activity of Nanoreactors by Biomimetic Engineering Polymer Membranes. Nano Lett. 2015, 15 (11), 7596-7603.

(33) Edlinger, C.; Einfalt, T.; Spulber, M.; Car, A.; Meier, W.; Palivan, C. G. Biomimetic Strategy To Reversibly Trigger Functionality of Catalytic Nanocompartments by the Insertion of PH-Responsive Biovalves. Nano Lett. 2017, 17 (9), 5790-5798.

(34) Macháň, R.; Wohland, T. Recent Applications of Fluorescence Correlation Spectroscopy in Live Systems. FEBS Lett. 2014, 588 (19), 3571-3584.

(35) Rigler, P.; Meier, W. Encapsulation of Fluorescent Molecules by Functionalized Polymeric Nanocontainers: Investigation by Confocal Fluorescence Imaging and Fluorescence Correlation Spectroscopy. J. Am. Chem. Soc. 2006, 128 (1), 367-373. 
(36) Martino, C.; deMello, A. J. Droplet-Based Microfluidics for Artificial Cell Generation: A Brief Review. Interface Focus 2016, 6 (4).

(37) Nishimura, K.; Matsuura, T.; Nishimura, K.; Sunami, T.; Suzuki, H.; Yomo, T. CellFree Protein Synthesis inside Giant Unilamellar Vesicles Analyzed by Flow Cytometry. Langmuir 2012, 28 (22), 8426-8432.

(38) Qiao, Y.; Li, M.; Booth, R.; Mann, S. Predatory Behaviour in Synthetic Protocell Communities. Nat. Chem. 2017, 9 (2), 110.

(39) Kita-Tokarczyk, K.; Grumelard, J.; Haefele, T.; Meier, W. Block Copolymer Vesicles-Using Concepts from Polymer Chemistry to Mimic Biomembranes. Polymer 2005, 46 (11), 3540-3563. 


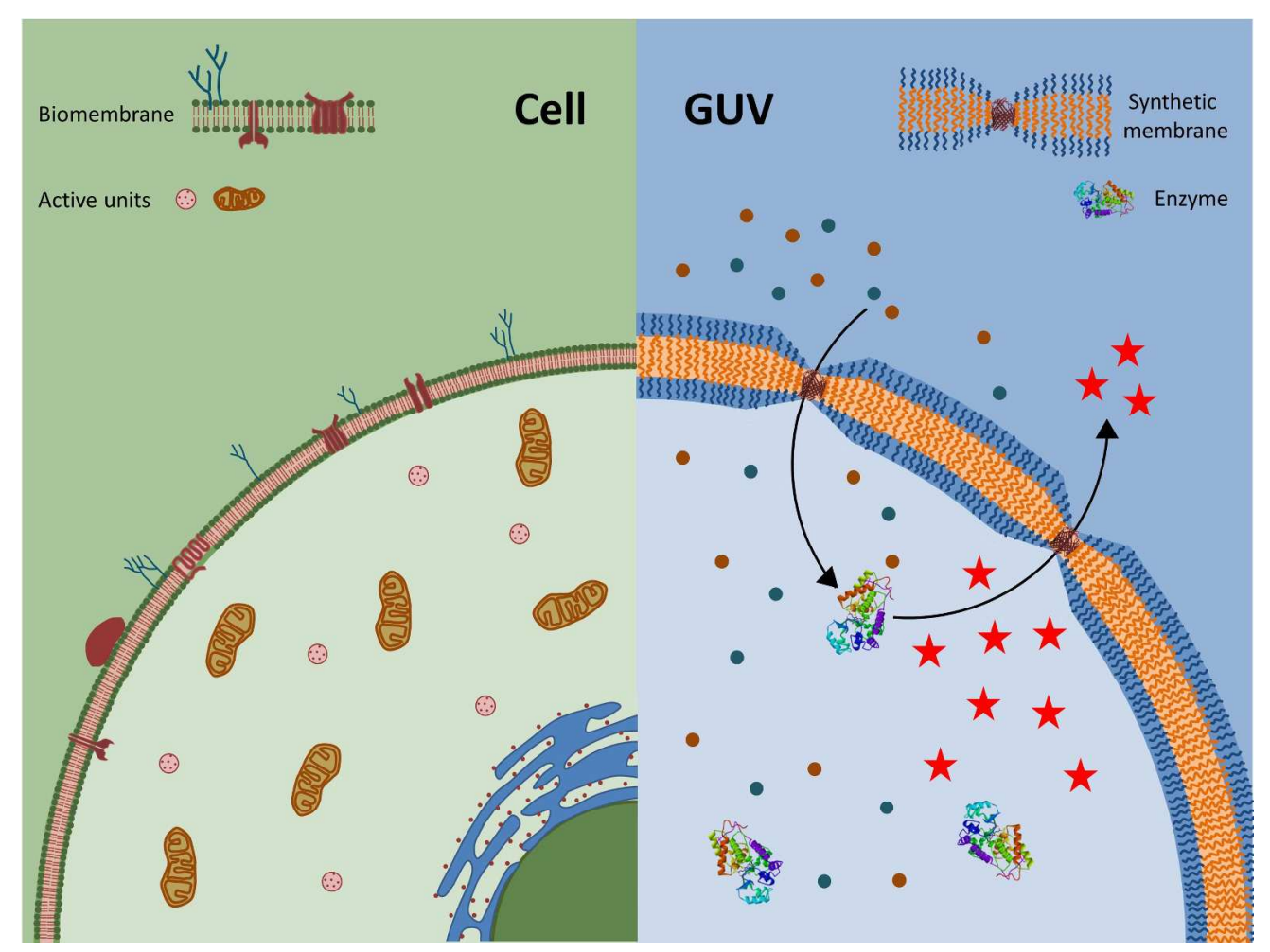

Figure 1

$381 \times 285 \mathrm{~mm}(200 \times 200$ DPI $)$ 

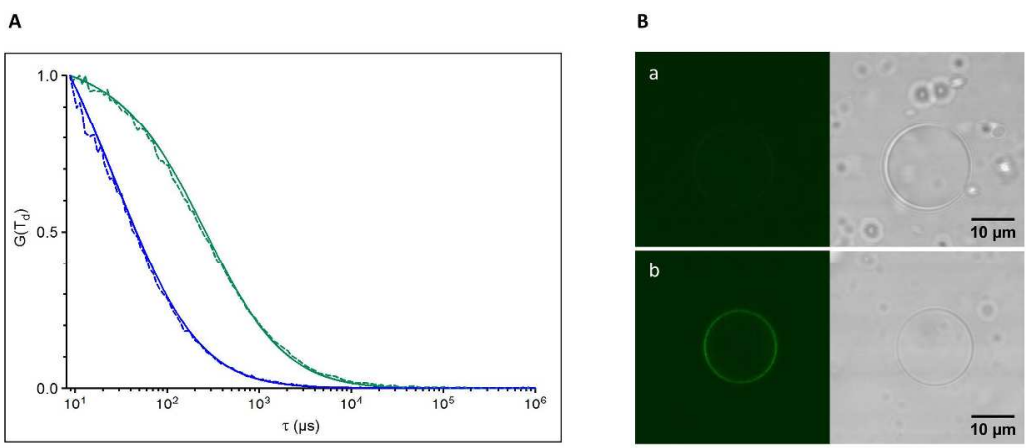

c

D
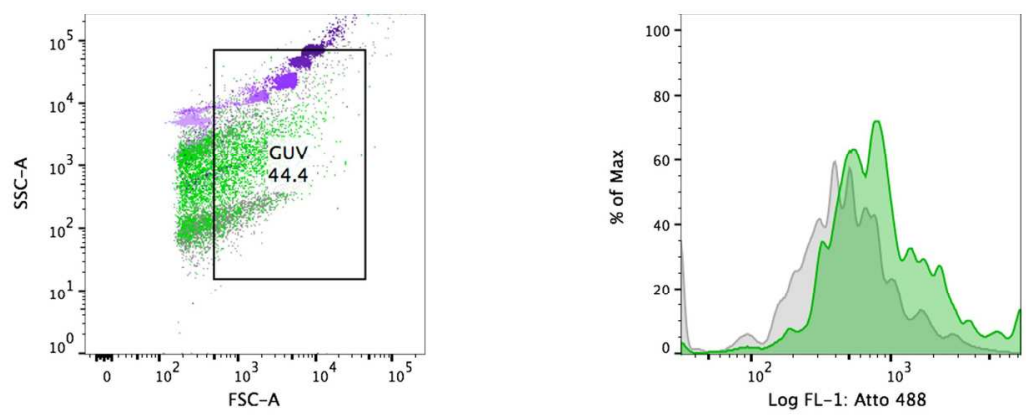

Figure 2

$381 \times 285 \mathrm{~mm}(200 \times 200$ DPI $)$

ACS Paragon Plus Environment 


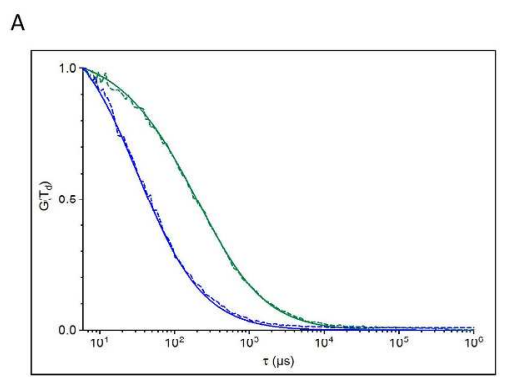

c
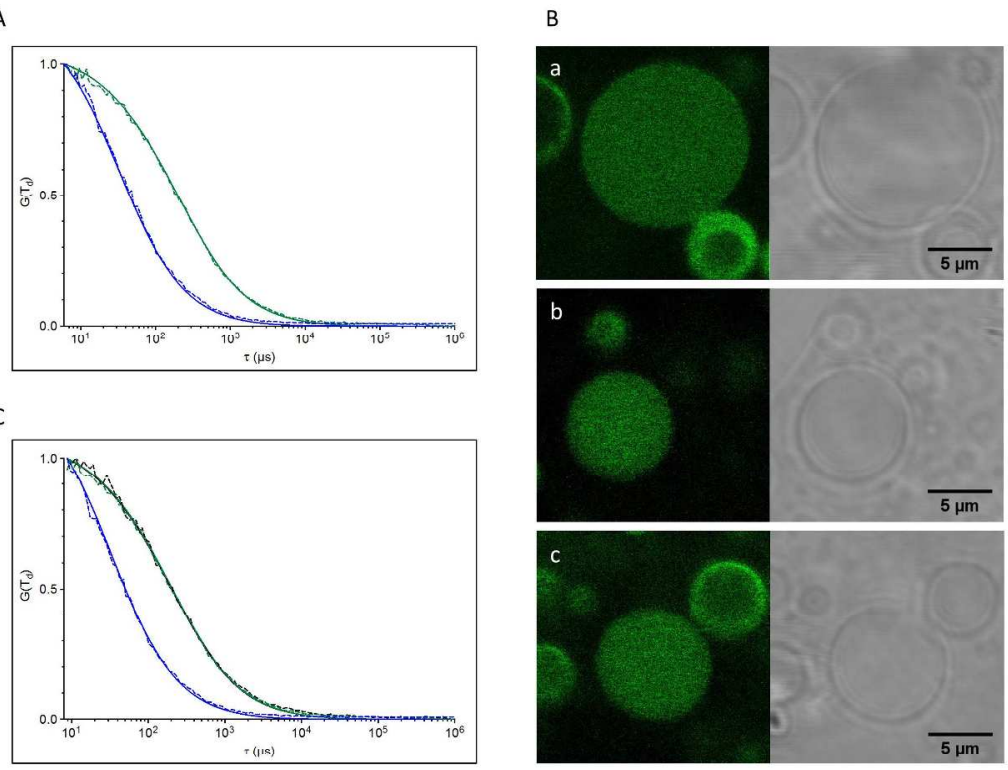

Figure 3

$381 \times 285 \mathrm{~mm}(200 \times 200$ DPI $)$

ACS Paragon Plus Environment 
GUV with reconstituted OmpF

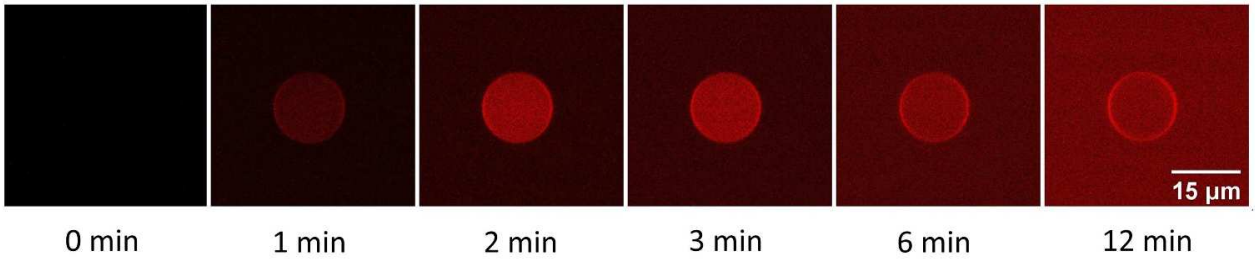

GUV without reconstituted OmpF

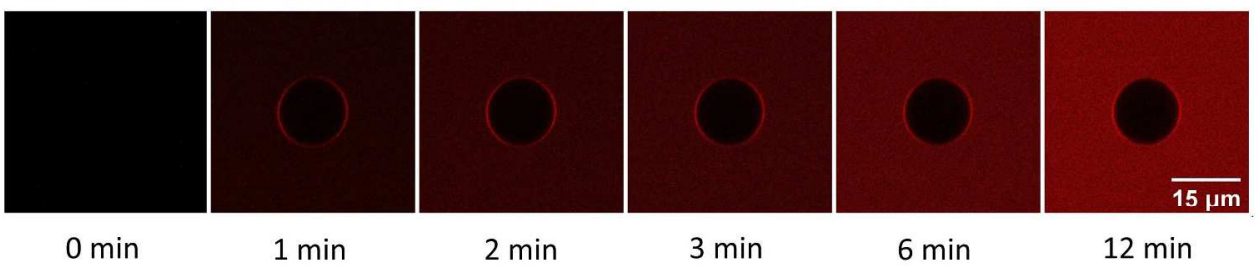

Figure 4

$381 \times 285 \mathrm{~mm}(200 \times 200$ DPI $)$ 
GUVs with reconstituted OmpF
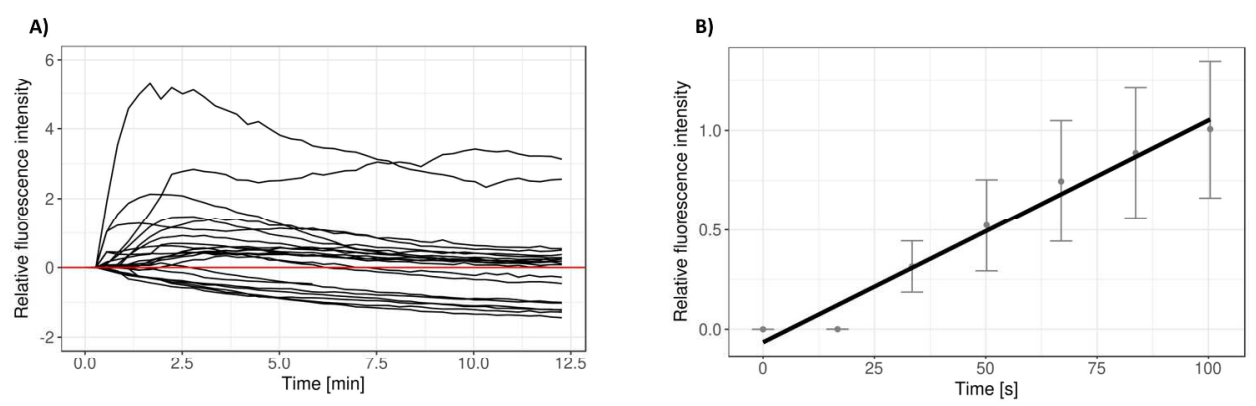

GUVs without reconstituted OmpF
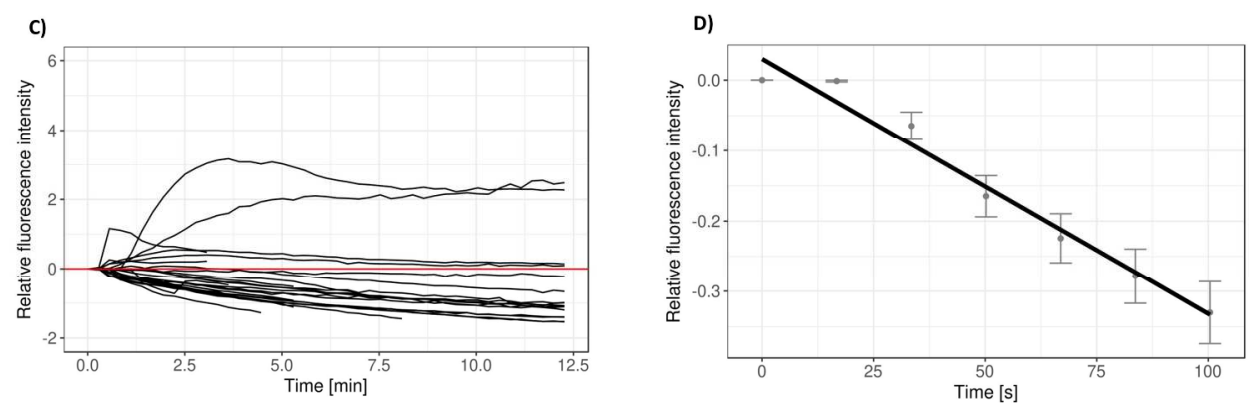

Figure 5

$381 \times 285 \mathrm{~mm}(200 \times 200$ DPI $)$ 


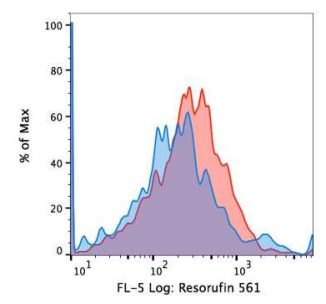

Figure 6 $381 \times 285 \mathrm{~mm}(200 \times 200$ DPI $)$ 
Conversion rate

A)

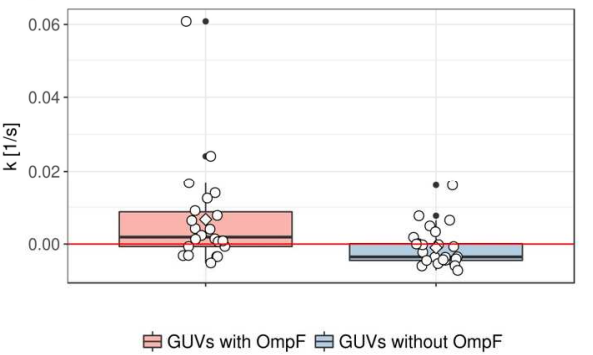

Conversion rate by size

B)

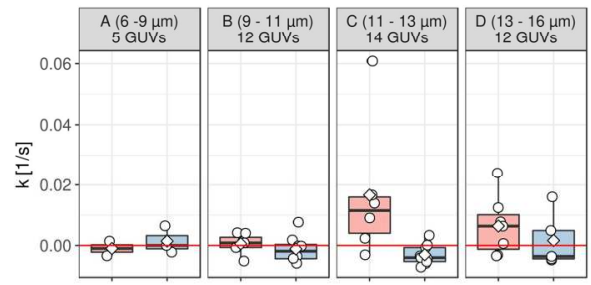

追 GUVs with OmpF 追 GUVs without OmpF

Figure 7

$381 \times 285 \mathrm{~mm}(200 \times 200$ DPI $)$

ACS Paragon Plus Environment 


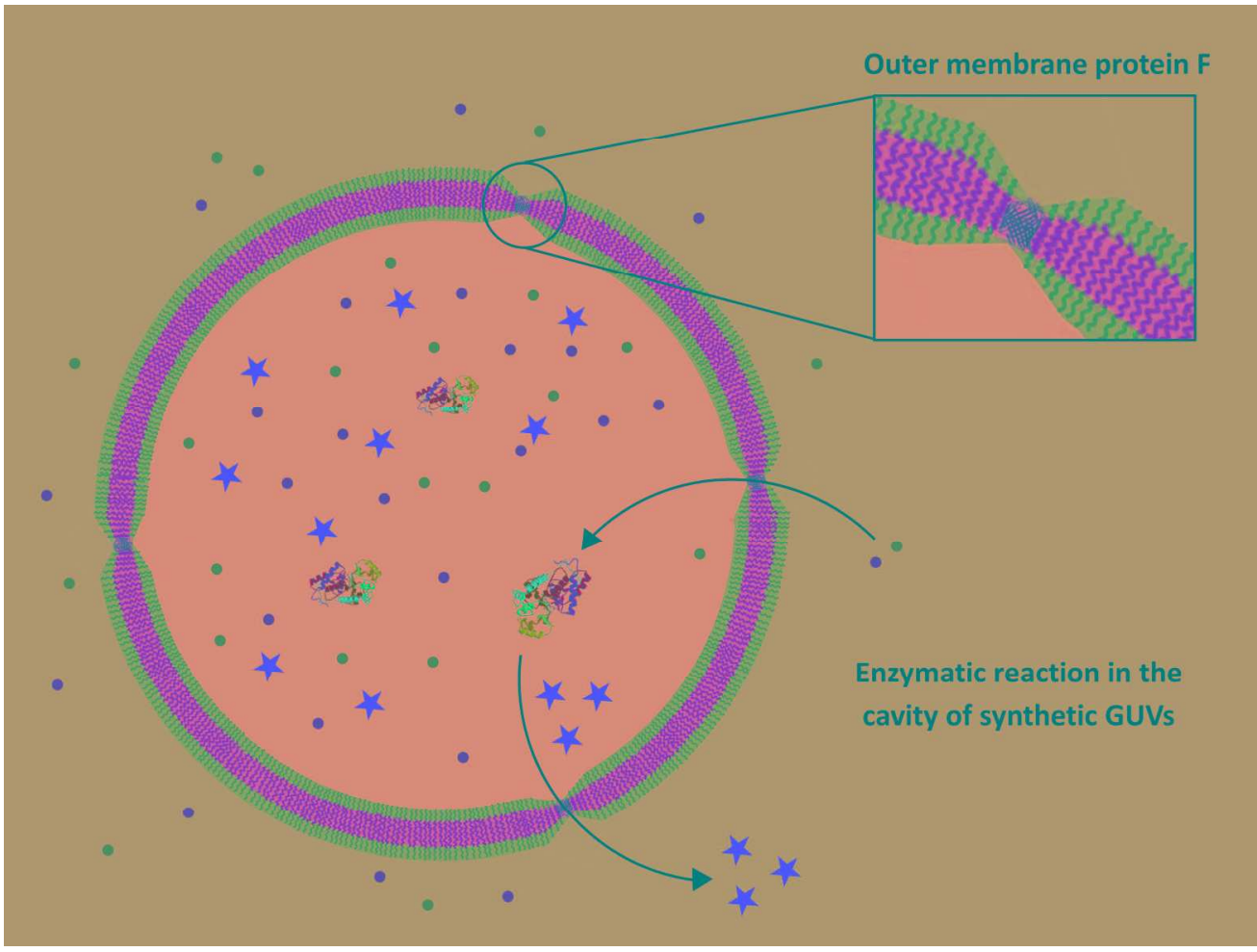

ToC Figure

$254 \times 190 \mathrm{~mm}(300 \times 300$ DPI)

ACS Paragon Plus Environment 